\title{
Loss of Krüppel-like factor 6 cripples podocyte mitochondrial function
}

\author{
Jeffrey B. Kopp \\ Kidney Diseases Branch, National Institute of Diabetes and Digestive and Kidney Diseases (NIDDK), NIH, Bethesda, Maryland, USA.
}

\begin{abstract}
Krüppel-like factors (KLFs) are zinc finger transcription factors that share homology in three C-terminal zinc finger domains. KLF family members are expressed in most if not all tissues and have diverse roles in organismal development and cell differentiation, function, and death. The glomerular podocyte is particularly sensitive to mitochondrial dysfunction, as seen in various genetic disorders manifesting as progressive glomerulosclerosis. In this issue of the $J \mathrm{Cl}$, Mallipattu and coworkers show that KLF6 expression is reduced in mouse and human glomerular disease. Podocyte-specific deletion of KIf6 expression in mice leads to mitochondrial dysfunction and apoptosis, followed by glomerulosclerosis. This is the first demonstration that defective transcriptional regulation of nuclear-encoded mitochondrial genes can result in experimental glomerular disease.
\end{abstract}

\section{Krüppel and Krüppel-like factors}

The 1995 Nobel Prize in Physiology or Medicine was award to Edward B. Lewis, Christiane Nüsslein-Volhard, and Eric F. Wieschaus for their work on embryonic development of Drosophila melanogaster, particularly with regard to homeotic genes that regulate development in plants and animals. Perhaps best known among the homeotic genes are those that encode the homeobox (Hox and ParaHox) proteins, which regulate other gene families (1). In 1950, Gloor described a fly mutant that caused severe body malformation, which he named Krüppel, meaning "cripple” (2). Nüsslein-Volhard and Wieschaus showed that Krüppel mutations disrupt Drosophila body segmentation at very early stages of embryogenesis, leading to embryonic lethality (3).

Mammalian genomes contain many genes that encode orthologs of Drosophila Krüppel, termed Krüppel-like factors (KLFs). KLFs manifest amino acid homology to Krüppel in the three DNA-binding zinc fingers located at the $\mathrm{C}$ terminus
(Figure 1). The highly conserved zinc finger domains comprise three sets of Cys2/His2 amino acid residues located at the $\mathrm{C}$ terminus, which are also similar to three motifs in the transcription factor SP1 (Figure 1). The zinc finger motifs of members of the KLF and SP1 superfamily of transcriptional regulators bind GC-rich motifs in gene regulatory regions, and they act as generally weak activators and often as strong repressors of gene expression. KLFs bound to DNA attract partners. For group 2 KLFs such as KLF4, which are transcriptional activators, these may include CBP/p300 (4). For example, KLF4 binds a TGF- $\beta$ transcriptional control element located in the angiotensin II type 1 receptor (ATR1). In response to TGF- $\beta$ signaling, SP1 dissociates from KLF4 and PPAR $\gamma$ binds KLF4, resulting in enhanced ATR1 transcription (5).

The human genome contains 17 KLF-encoding genes and can be classified into three phylogenetic groups that correlate with functional differences: group 1 KLFs are transcriptional repressors, group 2 KLFs are predominantly transcriptional

Related Article: p. 1347

Conflict of interest: The author has declared that no conflict of interest exists.

Reference information: J Clin Invest. 2015;125(3):968-971. doi:10.1172/JCI80280.

activators, and group $3 \mathrm{KLFs}$ bind and potentiate the activity of the co-repressor SIN3A; KLF16 and -17 do not cluster with other group members (ref. 6 and Figure 2). Expression of some KLFs is tissue restricted, such as KLF1 in hematopoietic cells, while others are widely expressed. This class of transcription factors has highly diverse roles that include regulating cell growth, differentiation, and death; endothelial and epithelial function; fat and glucose metabolism; and mitochondrial function; among other effects (6). Shinya Yamanaka and colleagues have shown that KLF4 is one of a set of four transcription factors (the others being OCT4, SOX2, and MYC) that can reprogram adult cells to become induced pluripotent stem cells (7), a discovery that resulted in Yamanaka sharing the 2012 Nobel Prize in Physiology or Medicine with John Gurdon. As transcriptional factors, KLFs are present in the nucleus, but they are also present in the cytoplasm and have distinct roles there.

\section{KLFs have diverse roles in kidney physiology and pathophysiology}

Glomerular endothelial cells that are exposed in vitro to laminar shear stress express higher levels of KLF2, leading Slater et al. to suggest that this might contribute to the phenotype-stabilizing effects of shear stress on the vascular endothelium (8). Compared with WT mice, animals with a endothelial-specific deletion of Klf2 manifest a more severe streptozotocin-diabetic nephropathy, including more proteinuria, more glomerular hypertrophy, and evidence of more endothelial injury but interestingly also more severe podocyte injury (9). In a study of glomerular transcripts expressed in atypical hemolytic uremic syndrome, KLF4 expression was modestly elevated (10); on the other hand, KLF2 and KLF4 transcripts were reduced in glomerular transcripts from renal transplant-associated thrombotic microangiopathy (11), suggesting a complex picture for KLF-mediated gene expression. 
A

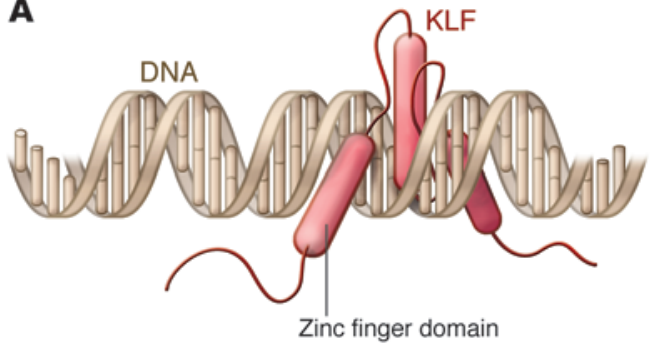

B

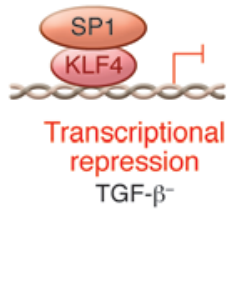

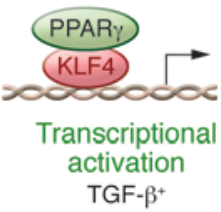

Figure 1. The KLF family of transcription factors. (A) KLF family members all contain three highly conserved zinc finger domains within their $C$ terminus that bind to $\mathrm{GC}$-rich regions in DNA. These three zinc finger domains insert into the major groove of the DNA helix. (B) KLF transcription factors generally bind proximal to the transcriptional start site, acting as transcriptional promoters. In the case of KLF4 bound to the angiotensin II receptor type 1 gene, the binding of SP1 confers inhibition of transcription. In the presence of TCF- $\beta$, SP1 dissociates from KLF4 and PPAR $\gamma$ binds, which promotes transcriptional initiation.

In contrast to the predominantly endothelial expression pattern of KLF2 and KLF4, KLF5 is expressed in collecting duct epithelial cells and plays a role in inflammation and fibrosis. Mice haploinsufficient for Klf5 exhibit less renal injury following unilateral ureteral ligation and an apparent shift from M1 macrophages to M2 macrophages, which would be compatible with a less fibrotic outcome (12).

During metanephric development, Klf6 is expressed in the Wolffian duct and its derivatives and in the ureteric bud and its branches, including the distal tubule, with limited glomerular staining seen in what was interpreted as mesangial cells (13). In diabetic Ren-2 rats on the other hand, Klf6 expression is increased in proximal tubular cells, and overexpression of KLF6 in cultured proximal tubular cells increased TGF- $\beta 1$ expression and promoted a phenotype suggestive of epithelial-mesenchymal transition (14). KLF12 is also expressed in the distal tubule, specifically in the inner medullary collecting tubule, and in vitro it was shown to drive expression of the urea transporter A-1 but not the epithelial sodium channel $(\mathrm{ENaC})(15)$.

KLF15 is decreased in the renal interstitium of $5 / 6$ nephrectomized rats and has been suggested to play an antifibrotic role, as in vitro studies demonstrate that KLF15 represses TGF- $\beta 1$ signaling via ERK/ MAPK and JNK/MAPK pathways (16). Two studies have addressed the role of KLF15 in glomerular cells. In the rat Thy1 nephritis model, glomerular KLF15 expression fell during the period of mesangial proliferation, and in cultured rat mesangial cells,

knockdown of KLF2 increased cell proliferation (17). In cultured human podocytes, retinoids were shown to increase KLF15 expression, and KLF15 drove podocyte differentiation in vitro, binding to the promoter regions of nephrin and podocin as shown by ChIP assay (18). Further, Klf15knockout mice manifest more podocyte injury in response to the podocyte toxins LPS and doxorubicin, suggesting a phenotype-stabilizing role for KLF15.

\section{KLFs and mitochondria}

KLFs have previously been associated with regulation of mitochondria number and function. Mitochondria are heterogeneous and dynamic organelles, with properties that may differ within a cell and between cells. For example, mitochondria differ in membrane potential and metabolic activity and in their tendency to be located in isolation or in aggregates with other mitochondria. In cultured pulmonary artery smooth muscle cells, KLF5 drives survivin expression, generates mitochondrial hyperpolarization, and protects against apoptotic cell death (19). In cultured neurites, KLF4 and KLF6 reduce mitochondrial complex size (isolated versus aggregated), and KLF6, but not KLF4, reduces mitochondrial density. As KLF6 is associated with increased neurite growth, it has been suggested that smaller, less dense mitochondria may participate in neurite growth (20). Finally, in Caenorhabditis elegans, Klf3 is essential for the expression of enzymes involved in beta-oxidation of fatty acids in mitochondria and peroxisomes (21).

As mitochondria are present in all nucleated cells, it is not surprising that genetic disorders that alter mitochondria can affect all tissues. With regard to kidney phenotypes, mitochondrial disorders may manifest as proximal tubular dysfunction with loss of electrolytes, glucose, amino acids, and low-molecular-weight proteins; tubulointerstitial disease and cystic kidney disease; and podocytopathy with subnephrotic or nephrotic proteinuria.

\section{Further insight into the role of KLF6 in podocyte biology}

In this issue, Mallipattu and coworkers present three lines of evidence that support a role for KLF6 in maintaining normal podocyte function and specifically link this to the ability of KLF6 to support podocyte mitochondrial function (22).

First, Mallipattu et al. surveyed renal expression of $9 \mathrm{KLFs}$ in HIV-infected human podocytes and in HIV-transgenic mice (23) that manifest with HIV-associated nephropathy (collapsing glomerulopathy). It is currently thought that podocyte injury and loss lead to podocyte replacement from several sources, particularly parietal epithelial stem cells and cells of the renin lineage. A survey of the mRNA expression patterns of KLF family members revealed that only KLF6 expression was altered, with it being reduced in both HIV-infected podocytes and in HIV transgenic mouse glomeruli.

Second, mice with podocyte-specific deletion of Klf6 manifested albuminuria but not glomerulosclerosis, as least within the observation period (22). Doxorubicin induces podocyte injury and results in glomerulosclerosis in susceptible mouse strains. Doxorubicin is also used in cancer chemotherapy, injuring or killing sensitive cells. While doxorubicin intercalates into DNA, the cytotoxic effects of this agent may be mediated by poisoning topoisomerase II, which corrects DNA supercoils that otherwise would result in tangled DNA, thereby releasing torsional stress; therefore, the expected result of decreased topoisomerase function would be compromised transcription (23). Doxorubicin-sensitive mice that manifest podocyte injury have a mutation in the DNA repair gene Prkdc (24). Further, doxorubicin decreases intracellular ATP (by mechanisms that remain unclear), resulting in a compensatory increase in mitochondrial 


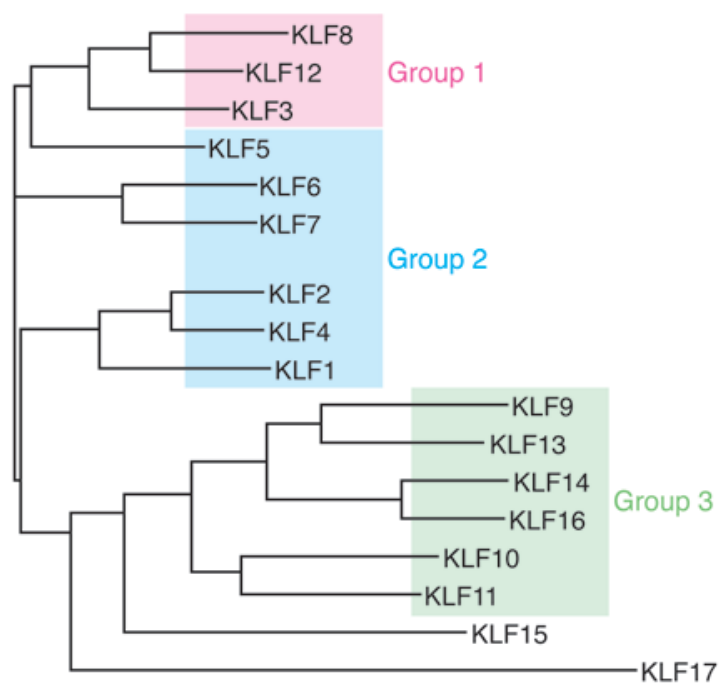

Figure 2. KLF phyologenetic tree. Analysis was conducted with full-length protein sequences using ClustalW to generate phylogenetic relationships between KLFs. Group 1 and 3 KLFs are transcriptional repressors, while group 2 KLFs are generally transcriptional activators. Figure adapted with permission from the American Physiological Society (6).

oxidation, and this may impose oxidative stress (25). This suggests that KLF6 contributes to progression of glomerular injury, at least in this model.

Third, Mallipattu and colleagues developed further in vivo and in vitro evidence that suggests KLF6 is essential for normal mitochondrial function, particularly in the setting of mitochondrial stress (22). Mice lacking Klf6 manifested dysmorphic mitochondria following doxorubicin exposure, and KLF6 expression increased in WT mice and in cultured mouse podocytes exposed to doxorubicin, suggesting a role in protection against mitochondrial stress. In cultured human podocytes, KLF6 knockdown was associated with decreased mitochondrial membrane potential, oxygen consumption rate, and ATP levels. Moreover, depletion of KLF6 was also associated with increased levels of cleaved caspase-3 and -9 , release of mitochondrial cytochrome $c$, and increased apoptosis.

Promoter analysis suggested that the human cytochrome $c$ oxidase assembly (SCO2) had KLF6-binding sites, which was confirmed by ChIP analysis. Furthermore, SCO2 expression was reduced in podocytes from KLF6-deficient mice. In human podocytes, knockdown of SCO2 expression increased cleaved caspase-9 levels and release of cytochrome $c$, adding some support to the proposal by Mallipattu and colleagues that SCO2 may be a key KLF6 target for mediating protection against mitochondrial injury (22). The expression of a number of other mitochondrial genes was also reduced in podocyte-specific Klf6-knockout mice, including nuclear respiratory factor 1 (Nrfl) and mitochondrial inner membrane protein (Mpv17); however, it remains to be determined whether these genes are directly or indirectly affected by KLF6 deficiency.

\section{Mitochondria and podocyte function}

When one looks at a kidney biopsy under the electron microscope, it is apparent that the renal cells endowed with the most mitochondria are proximal tubular cells, which depend heavily on mitochondrial oxidative phosphorylation to support sodium transport. Nevertheless, it is striking that genetic disorders that affect mitochondrial function often include podocyte dysfunction as part of a spectrum of cellular pathologies (Table 1). Mitochondrial function can be disrupted by mutations in nuclear-encoded genes and mitochondrial DNA-encoded genes, as has been the subject of a recent comprehensive review (26). Within the kidney, mutations that affect mitochondrial-expressed genes tend to manifest primarily as tubular epithelial cell dysfunction or podocyte dysfunction.

The apparent high-energy requirement of the podocyte is not well understood but has been remarked upon by several groups (27-29). Possible reasons include foot process motility, which is enabled by continuous remodeling of the actin cytoskeleton; uptake of filtered proteins; or other unknown mechanisms. Another link between energy shortage and podocyte injury is the observation that defective and damaged mitochondria are cleared by autophagy, a process linked to activation of inflammasomes and apoptosis (30). Therapeutic strategies to address podocyte mitochondrial dysfunction hold considerable promise.

\section{Table 1. Overview of genetic mutations associated with podocyte dysfunction}

\begin{tabular}{|c|c|c|}
\hline Manifestation & Nuclear genes & Mitochondrial DNA-encoded genes \\
\hline Tubular dysfunction is prominent, typically spares the podocyte & $\begin{array}{l}\text { OX-PHOS defects: mutations in the genes affecting } \\
\text { complex III and IV are more common than complex I }\end{array}$ & mtDNA: often large deletions \\
\hline & Fatty acid metabolism defects: CPT1 & \\
\hline Podocyte dysfunction is typically present & $\begin{array}{c}\text { Human: COQ10 synthesis - } \mathrm{COQ2} \text {, COQ6, } \\
\text { COQ9, PDSS2, ADCK4 } \\
\text { Mouse model: Mpv17, KIf6 }\end{array}$ & MELAS: tRNA-Leu \\
\hline
\end{tabular}

For a comprehensive list of genes associated with kidney disease, see ref. 24. OX-PHOS, the proteins associated with oxidative phosphorylation; COQ10, coenzyme Q10 (ubiquinone); MELAS, mitochondrial encephalopathy, lactic acidosis, and stroke-like episodes. 
For example, therapy with the electron transport chain component coenzyme Q10 (COQ10) can reduce progressive renal damage in individuals with mutations in genes that affect the COQ10 synthetic pathway, providing a strong rationale for genetic testing of patients with nephrotic syndrome, particularly children, to identify individuals who could benefit from this therapy (31). Other new therapeutic approaches are also being explored. For example, PPAR $\alpha$ protects podocytes from aldosterone-induced mitochondrial dysfunction (32). Glycogen synthetase kinase (GSK) $3 \beta$ integrates signals that regulate mitochondrial pore transition, and a thiazolidinedione compound that inhibits GSK2 $\beta$ was shown to alleviate doxorubicin-induced podocyte injury in vitro and in vivo (33).

The study by Mallipattu and colleagues gives one more example of why this is an exciting time in glomerular pathophysiology, as existing concepts are being remodeled by new findings obtained from human genetic and pathology studies, animal and cell culture models, and systems biology approaches applied to large patient cohorts.

\section{Acknowledgments}

This work was supported by the Intramural Research Program of the NIDDK, NIH. The author wishes to acknowledge the generosity of Beth McConnell and colleagues in allowing the adaptation of Figure 2, and the help of Jurgen Heymann with manuscript review.

Address correspondence to: Jeffrey Kopp, 10 Center Drive, 3N116, NIH, Bethesda, Maryland 20192-1268, USA. Phone: 301.594.3403; E-mail: jbkopp@nih.gov.

1. Gehring WJ, Kloter U, Suga H. Evolution of the Hox gene complex from an evolutionary ground state. Curr Top Dev Biol. 2009;88:35-61.

2. Gloor H. Schädigungsmuster eines Letalfaktors (Kr) von Drosophila melanogaster. Arch Jul Klaus Stiftung. 1950;25:38-44.

3. Wieschaus E, Nusslein-Volhard C, Kluding H. Kruppel, a gene whose activity is required early in the zygotic genome for normal embryonic segmentation. Dev Biol. 1984;104(1):172-186.

4. Mas C, et. al. Structural and functional characterization of an atypical activation domain in erythroid Krüppel-like factor (EKLF). Proc Natl Acad Sci U S A. 2011;108(26):10484-10489.

5. Zhang XH, Zheng B, Gu C, Fu JR, Wen JK. TGF- $\beta 1$ downregulates AT1 receptor expression via $\mathrm{PKC}-\delta$-mediated $\mathrm{Sp} 1$ dissociation from KLF4 and Smad-mediated PPAR- $\gamma$ association with KLF4. Arterioscler Thromb Vasc Biol. 2012;32(4):1015-1023.

6. McConnell BB, Yang VW. Mammalian Krüppellike factors in health and diseases. Physiol Rev. 2010;90(4):1337-1381.

7. Takahashi K, Yamanaka S. Induction of pluripotent stem cells from mouse embryonic and adult fibroblast cultures by defined factors. Cell. 2006;126(4):663-676.

8. Slater SC, et al. Chronic exposure to laminar shear stress induces Kruppel-like factor 2 in glomerular endothelial cells and modulates interactions with co-cultured podocytes. Int J Biochem Cell Biol. 2012;44(9):1482-1490.

9. Zhong F, et al. Reduced Kruppel-like factor 2 expression may aggravate the endothelial injury of diabetic nephropathy [published online ahead of print September 3, 2014]. Kidney Int. doi:10.1038/ki.2014.286.

10. Modde F, et al. Comprehensive analysis of glomerular mRNA expression of pro- and antithrombotic genes in atypical haemolytic-uremic syndrome (aHUS). Virchows Arch. 2013;462(4):455-464.

11. Agustian PA, et al. Glomerular mRNA expression of prothrombotic and antithrombotic factors in renal transplants with thrombotic microangiopathy. Transplantation. 2013;95(10): 1242-1248.

12. Fujiu K, Manabe I, Nagai R. Renal collecting duct epithelial cells regulate inflammation in tubulointerstitial damage in mice. J Clin Invest. 2011;121(9):3425-3441.

13. Fischer EA, Verpont MC, Garrett-Sinha LA, Ronco PM, Rossert JA. Klf6 is a zinc finger protein expressed in a cell-specific manner during kidney development. JAm Soc Nephrol. 2001;12(4):726-735.

14. Holian J, et al. Role of Kruppel-like factor 6 in transforming growth factor- $\beta 1$-induced epithelial-mesenchymal transition of proximal tubule cells. Am J Physiol Renal Physiol. 2008;295(5):F1388-F1396.

15. Suda S, Rai T, Sohara E, Sasaki S, Uchida S. Postnatal expression of KLF12 in the inner medullary collecting ducts of kidney and its trans-activation of UT-A1 urea transporter promoter. Biochem Biophys Res Commun. 2006;344(1):246-252.

16. Gao X, Wu G, Gu X, Fu L, Mei C. Kruppel-like factor 15 modulates renal interstitial fibrosis by ERK/MAPK and JNK/MAPK pathways regulation. Kidney Blood Press Res. 2013;37(6):631-640. 17. Hong Q, et al. Kruppel-like factor-15 inhibits the proliferation of mesangial cells. Cell Physiol Biochem. 2012;29(5):893-904.

18. Mallipattu SK, et al. Kruppel-like factor 15 (KLF15) is a key regulator of podocyte differentiation. J Biol Chem. 2012;287(23):19122-19135.

19. Courboulin A, et al. Kruppel-like factor 5 contributes to pulmonary artery smooth muscle proliferation and resistance to apoptosis in human pulmonary arterial hypertension. Respir Res. 2011;12:128.

20. Steketee MB, et al. Mitochondrial dynamics regulate growth cone motility, guidance, and neurite growth rate in perinatal retinal ganglion cells in vitro. Invest Ophthalmol Vis Sci. 2012;53(11):7402-7411.

21. Zhang J, et al. Regulation of fat storage and reproduction by Kruppel-like transcription factor KLF3 and fat-associated genes in Caenorhabditis elegans. J Mol Biol. 2011;411(3):537-553.

22. Mallipattu SK, et al. Krüppel-like factor 6 regulates mitochondrial function in the kidney. J Clin Invest. 2015;125(3):1347-1361.

23. Yang F, Teves SS, Kemp CJ, Henikoff S. Doxorubicin, DNA torsion, and chromatin dynamics. Biochim Biophys Acta. 2014;1845(1):84-89.

24. Papeta N, et al. Prkdc participates in mitochondrial genome maintenance and prevents Adriamycin-induced nephropathy in mice. J Clin Invest. 2010;120(11):4055-4064.

25. Strigun A, Wahrheit J, Niklas J, Heinzle E, Noor F. Doxorubicin increases oxidative metabolism in HL-1 cardiomyocytes as shown by ${ }^{13} \mathrm{C}$ metabolic flux analysis. Toxicol Sci. 2012;125(2):595-606.

26. O'Toole JF. Renal manifestations of genetic mitochondrial disease. Int J Nephrol Renovasc Dis. 2014;7:57-67.

27. Imasawa T, Rossignol R. Podocyte energy metabolism and glomerular diseases. Int J Biochem Cell Biol. 2013;45(9):2109-2118.

28. Muller-Deile J, Schiffer M. The podocyte power-plant disaster and its contribution to glomerulopathy. Front Endocrinol (Lausanne). 2014;5:209.

29. Yan K, et al. The struggle for energy in podocytes leads to nephrotic syndrome. Cell Cycle. 2012;11(8):1504-1511.

30. Fougeray S, Pallet N. Mechanisms and biological functions of autophagy in diseased and ageing kidneys. Nat Rev Nephrol. 2015;11(1):34-45.

31. Ashraf S, et al. ADCK4 mutations promote steroid-resistant nephrotic syndrome through CoQ10 biosynthesis disruption. J Clin Invest. 2013;123(12):5179-5189.

32. Zhu C, et al. Mitochondrial dysfunction mediates aldosterone-induced podocyte damage: a therapeutic target of PPAR $\gamma$. Am J Pathol. 2011;178(5):2020-2031.

33. Wang Z, et al. Pharmacological targeting of GSK3 $\beta$ confers protection against podocytopathy and proteinuria by desensitizing mitochondrial permeability transition. Br J Pharmacol. 2015;172(3):895-909. 